\title{
Cholinergic Regulation of Airway Inflammation and Remodelling
}

\author{
Saeed Kolahian' ${ }^{1}$ and Reinoud Gosens ${ }^{2}$ \\ ${ }^{1}$ Department of Basic Sciences, Faculty of Veterinary Medicine, University of Tabriz, Iran \\ ${ }^{2}$ Department of Molecular Pharmacology, University of Groningen, Antonius Deusinglaan 1, 9713 AV Groningen, The Netherlands
}

Correspondence should be addressed to Reinoud Gosens, r.gosens@rug.nl

Received 21 June 2011; Accepted 10 October 2011

Academic Editor: Irene Heijink

Copyright (C) 2012 S. Kolahian and R. Gosens. This is an open access article distributed under the Creative Commons Attribution License, which permits unrestricted use, distribution, and reproduction in any medium, provided the original work is properly cited.

Acetylcholine is the predominant parasympathetic neurotransmitter in the airways that regulates bronchoconstriction and mucus secretion. Recent findings suggest that acetylcholine regulates additional functions in the airways, including inflammation and remodelling during inflammatory airway diseases. Moreover, it has become apparent that acetylcholine is synthesized by nonneuronal cells and tissues, including inflammatory cells and structural cells. In this paper, we will discuss the regulatory role of acetylcholine in inflammation and remodelling in which we will focus on the role of the airway smooth muscle cell as a target cell for acetylcholine that modulates inflammation and remodelling during respiratory diseases such as asthma and COPD.

\section{Introduction}

Acetylcholine is classically viewed as a neurotransmitter that regulates cognitive and behavioural functions in the brain, autonomous ganglionic transmission, and parasympathetic postganglionic transmission. In the respiratory tract, acetylcholine is the predominant parasympathetic neurotransmitter and its role in the regulation of bronchomotor tone and mucus secretion from airway submucosal glands is well established [1]. More recent findings suggest that acetylcholine regulates additional functions in the respiratory tract, including inflammation and remodelling during inflammatory lung diseases [2-4]. Moreover, it has become apparent that acetylcholine is synthesized by nonneuronal cells and tissues, particularly inflammatory cells and the airway epithelium [5-7]. These cells also express receptors for acetylcholine, including muscarinic receptors and nicotinic receptors that modulate inflammatory responses $[2,6]$. Collectively, these findings have questioned the traditional view on the physiological and pathophysiological role of acetylcholine, which has opened up new possibilities for therapeutic targeting of the pulmonary cholinergic system. In this paper, we will discuss these recent findings in which we will focus on the role of the airway smooth muscle cell as a target for acetylcholine in inflammation and remodelling during respiratory diseases such as asthma and COPD.

\section{The Origin of Acetylcholine}

Acetylcholine is biosynthesized from choline and acetyl-CoA by choline acetyltransferase (ChAT) or carnitine acetyltransferase (CarAT) by several cell types in the respiratory tract [6]. Airway neurons and airway epithelial cells express ChAT and have been demonstrated by HPLC detection to release acetylcholine [5]. The release of acetylcholine from other nonneuronal tissues in the respiratory tract is suggested by the fact that also macrophages, mast cells, fibroblasts, smooth muscle cells, lymphocytes, and granulocytes express ChAT immunoreactivity [6]; however the release of acetylcholine from these cells and tissues has not yet been measured directly in the respiratory tract. Acetylcholine exerts its functions either via muscarinic receptors, a class of G-proteincoupled receptor subtypes, or via nicotinic receptors, a class of ligand-gated cation channels [8]. Most structural cells and inflammatory cells that are present in the respiratory system, including smooth muscle cells, fibroblasts, epithelial cells, mast cells, granulocytes, lymphocytes, and macrophages, express muscarinic and/or nicotinic receptors $[2,6]$. For 
a detailed overview of individual receptor subtypes and subunits expressed by these cells, we refer to a recent excellent overview by Wessler and Kirkpatrick [6]. The expression of muscarinic and nicotinic receptors, the expression of synthesizing enzymes such as ChAT, and the direct measurement by HPLC detection of acetylcholine release from nonneuronal tissues and cell cultures are solid evidence for the existence of a nonneuronal cholinergic system in addition to the more established neuronal cholinergic system in the airways.

The processing of acetylcholine by nonneuronal cells and tissues is not yet described in full although, for airway epithelial cells, secretory mechanisms have been described. Airway epithelial cells express the high affinity choline transporter (CHT1) that is involved in choline uptake as well as the organic cation transporter (OCT) subtypes 1 and 2, which play a dominant role in the release of acetylcholine by airway epithelial cells $[9,10]$. Furthermore, the expression of the vesicular acetylcholine transporter (VAChT) by epithelial cells has been reported suggesting that storage of acetylcholine in vesicles and release via the fusion of these vesicles with the plasma membrane, as occuring in neurons, may represent an additional mechanism for acetylcholine release by nonneuronal cell types $[9,10]$.

The breakdown of acetylcholine into acetic acid and choline is catalysed by acetylcholinesterase (AChE) and butyrylcholinesterase (BuChE), also known as pseudocholinesterase. The functional expression of AChE by airway epithelial cells is evidenced by observations that acetylcholine concentrations in cell supernatants of airway epithelial cell cultures were enhanced by the pharmacological inhibitor of AChE, neostigmine [5]. Collectively, the above-mentioned observations indicate that both neurons and nonneuronal cells and tissues in the respiratory system express and release acetylcholine. The functional role of nonneuronal acetylcholine on the airway smooth muscle includes bronchoconstriction [11, 12]. Additionally, acetylcholine may modulate airway hyperresponsiveness and remodelling, including the regulation of airway smooth muscle growth and the regulation of airway inflammation that promotes hyperresponsivness and remodelling. This role for acetylcholine will be discussed in the following sections.

\section{The Muscarinic Receptor: Acetylcholine as a Proinflammatory and Remodelling Mediator}

Muscarinic receptors are expressed by most structural cells in the airway wall, including the airway smooth muscle and by inflammatory cells that are involved in the pathogenesis of obstructive airway diseases [2]. Muscarinic receptors appear to play a proinflammatory role on these cells, suggesting that inhibition of muscarinic receptor function may have antiinflammatory effects in these diseases. Increased expression of muscarinic $M_{1}$ and $M_{3}$ receptors on airway structural cells and sputum cells of COPD patients has been reported $[13,14]$. Likewise, reduced expression of the autoinhibitory $\mathrm{M}_{2}$ receptor on airway neurons in asthma has been reported [1]. Both effects could contribute to enhanced acetylcholine release and function in these diseases. The proinflammatory role of acetylcholine via muscarinic receptors is discussed below.

\subsection{Direct Effects of Acetylcholine on Airway Smooth Muscle.} The airway smooth muscle expresses muscarinic $\mathrm{M}_{2}$ and $\mathrm{M}_{3}$ receptors roughly in a 4:1 ratio [15]. The muscarinic $\mathrm{M}_{3}$ receptor represents a primary target of acetylcholine in the airways, involved in the regulation of bronchoconstriction [15-18]. In addition, muscarinic receptors regulate proliferative and proinflammatory functions of the airway smooth muscle. It was observed that coadministration of muscarinic agonists with epidermal growth factor (EGF) in human airway smooth muscle cells induces a synergistic proliferative stimulus. This effect was associated with sustained activation of p70 S6 kinase $[19,20]$, an effect mediated by $\mathrm{G}_{\mathrm{q}}$ derived $\mathrm{G}_{\beta \gamma}$ subunits that activate phosphatidylinositol-3kinase (PI3K) in concert with the EGF receptor $[19,21]$. In line with these findings, muscarinic receptor agonists induce an increase in proliferation of airway smooth muscle cells in combination with platelet-derived growth factor (PDGF) [22], which is mediated by $\mathrm{G}_{\mathrm{q}}$-protein-coupled muscarinic $\mathrm{M}_{3}$ receptors and appears to involve a synergistic inhibitory phosphorylation of glycogen synthase kinase-3 (GSK-3) [23]. GSK-3 is a multitasking enzyme that regulates multiple signalling proteins and transcription factors involved in contractile protein expression and cell proliferation of airway smooth muscle [23-26].

Muscarinic-receptor-induced airway remodelling could also involve mechanical regulation as airway smooth muscle constriction results in airway epithelial cell compression and subsequent activation of EGFR phosphorylation in the airway epithelium [27]. Indeed, a recent clinical trial demonstrates that repeated methacholine inhalations cause airway remodelling in the absence of inflammation, characterized by collagen deposition and increased TGF- $\beta 1$ expression [28]. It is not yet clear whether such effects could also directly regulate remodelling of airway smooth muscle; however, mechanical strain of airway smooth muscle regulates cell proliferation and contractile protein expression [29-31], an effect enhanced in the presence of carbachol [32]. Clearly, this hypothesis needs to be followed up in future studies.

Muscarinic receptors on airway smooth muscle cells could also play a profound role in regulating the immunomodulatory function of airway smooth muscle $[33,34]$. Cholinergic stimulation with the muscarinic receptor agonist carbachol augments inflammatory gene expression in bovine tracheal smooth muscle in combination with cyclic stretch, which induces a synergistic increase in the expression of IL-6, IL-8, cyclo-oxygenase (COX) 1 and 2, and urokinasetype plasminogen activator (PLAU) [35]. It was recently demonstrated that the activation of muscarinic receptors also interacts with several cytokines and growth factors that play an important role in the pathogenesis of asthma and COPD, in particular with TNF- $\alpha$, PDGF-AB and cigarette smoke to enhance their inflammatory response in airway smooth muscle cells [36]. Thus, muscarinic $\mathrm{M}_{3}$ receptor stimulation of airway smooth muscle with methacholine induces IL- 6 and IL- 8 production and augments the release 
of these cytokines induced by cigarette smoke extract [36]. Our unpublished data show that this effect is dependent on downstream signalling to PKC, which activates the $\mathrm{I} \kappa \mathrm{B} \alpha / \mathrm{NF}-\kappa \mathrm{B}$ and MEK/ERK1/2 pathways [37]. This indicates that acetylcholine may also play an important role in the immunomodulatory processes driven by human airway smooth muscle.

The functional importance of these in vitro findings is illustrated by our in vivo studies that indicate a protective role for tiotropium bromide, a long-acting muscarinic antagonist, in the progression of airway smooth muscle remodelling. Thus, guinea pigs challenged with allergen for 12 consecutive weeks developed increased airway smooth muscle mass, increased contractile protein expression, and increased airway smooth muscle contractility, which were partially to fully prevented by treatment with tiotropium bromide [38]. In part, these inhibitory effects may have been due to the anti-inflammatory properties of tiotropium as airway eosinophilia was almost completely reduced by treatment with this compound [39]. Mucus gland remodelling and MUC5A/C hypersecretion were also prevented [39]. These results indicate that acetylcholine plays an essential role in remodelling of the airway smooth muscle (Figure 1). These effects may be direct, as suggested by the in vitro studies mentioned above, or indirect, as illustrated below.

3.2. Additional Effects of Acetylcholine on Airway Remodelling. Airway smooth muscle cells are embedded in the airway wall, and bidirectional communication between the muscle layer and the cell types and matrix protein structures that surround the muscle bundle is key to the development of abnormalities in airway smooth muscle phenotype and function in obstructive airways disease [40]. Fibroblasts are key effector cells in the production of extracellular matrix proteins that surround the airway smooth muscle bundle in the adventitia and submucosa of the airway wall [41]. Fibroblasts express functional muscarinic $\mathrm{M}_{2}$ and $\mathrm{M}_{3}$ receptors (predominantly $\mathrm{M}_{2}$ receptors with relatively fewer $\mathrm{M}_{3}$ receptors) [42]. In vitro, the muscarinic agonists carbachol and oxotremorine cause an increase in $\left({ }^{3} \mathrm{H}\right)$-thymidine incorporation (as a measure of cell proliferation) in human lung fibroblast cell lines and primary fibroblasts. This effect is mediated by the $\mathrm{M}_{2}$ receptor and regulated by the MEK/ERK1/2 pathway [42, 43]. Tiotropium, a long-acting muscarinic antagonist, concentration-dependently inhibited ACh-induced proliferation of primary human fibroblast isolated from biopsies of lung fibrosis patients and myofibroblasts derived from these cells [44]. Furthermore, it was found that muscarinic agonists stimulate the incorporation of ${ }^{3} \mathrm{H}$-proline into cellular proteins (as a measure of collagen synthesis) in human lung fibroblast cell lines and primary fibroblasts [45]. Also, tiotropium bromide inhibits collagen expression in the lung and small airways in guinea pigs repeatedly exposed to LPS [46]. Collectively, these studies support a role for acetylcholine in regulating fibroblast cell responses associated with remodelling.

A proinflammatory role of acetylcholine in fibroblasts was recently questioned by a study showing that the release of chemotactic mediators was not induced in fibroblasts incubated with acetylcholine because of a relative lack of $\mathrm{M}_{3}$ receptor expression in these cells [47]. On the other hand, primary lung fibroblast cultures from surgical specimens of COPD patients treated with acetylcholine showed enhanced IL- 8 and matrix metalloproteinase- 2 release. This effect was mediated by muscarinic $\mathrm{M}_{3}$ receptors [14], and tiotropium has an attenuating effect on metalloproteinase2 production from lung fibroblasts induced by inflammatory stimulation [48]. It is possible that the enhanced expression of muscarinic receptors by fibroblasts of COPD patients explains the discrepancy between these two studies as Profita et al. [14] showed that muscarinic $M_{1}$ and $M_{3}$ receptor as well as ChAT expressions were increased in fibroblasts from COPD patients. Although the quantification of muscarinic receptor expression using antibodies should be approached with care [49], functional differences between healthy controls and COPD patients were also observed. In this study, acetylcholine induced a significant increase in the activation of the ERK1/2 and NF $\kappa$ B pathways in fibroblasts of patients with COPD and promoted cell proliferation to a greater extent than observed in fibroblasts of healthy controls [14]. These findings clearly indicate the function of fibroblasts in remodelling processes that occur in chronic inflammatory airway diseases but the proinflammatory role of lung fibroblasts in response to acetylcholine remains to be studied in further detail.

The airway epithelium is key to the development of airway inflammation and remodelling as it presents the first barrier to inhaled particles and allergens and regulates the secretion of proinflammatory cytokines. Epithelial damage during allergic airway inflammation plays a key role in asthma and exposes sensory nerve endings in the submucosa to the airway lumen, which promotes reflex mechanisms leading to enhanced vagal release of acetylcholine [40]. Moreover, the airway epithelium is predominant in its expression of ChAT and may present a direct source of nonneuronal acetylcholine [5]. Acetylcholine is a proliferative stimulus for human bronchial epithelial cells in culture in part by activation of muscarinic $\mathrm{M}_{1}$ receptors [50,51]. Acetylcholine also increased eosinophil, monocyte, and neutrophil chemotactic activity by bronchial epithelial cells [52, 53]. This effect probably involves muscarinic $M_{1}$ receptors that induce leukotriene $\mathrm{B}_{4}$ release from epithelial cells, which in turn stimulates eosinophil, neutrophil, and monocyte chemotactic activities $[52,53]$. Muscarinic receptor agonists also induced the release of prostanoids from airway epithelial cells. Thus, muscarinic $\mathrm{M}_{3}$ receptors promote the activation of phospholipase A2, which stimulates the release of $\mathrm{PGE}_{2}$ from isolated tracheae, but only in preparations with an intact epithelial layer [54]. In addition, a recent investigation in human bronchial epithelial cells showed that acetylcholine induces the production of IL-8, involving PKC, ERK1/2, and $\mathrm{NF} \kappa \mathrm{B}$ pathway activation via muscarinic receptors [55].

Collectively, these findings indicate that acetylcholine, derived from the vagal nerve and from nonneuronal origins such as the airway epithelium, may induce cell responses associated with airway wall remodelling and trigger proinflammatory cytokine release by structural cells of the airway wall, including airway epithelial cells, airway fibroblasts, and 
Healthy airway

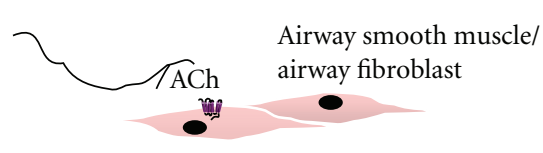

Inflamed airway

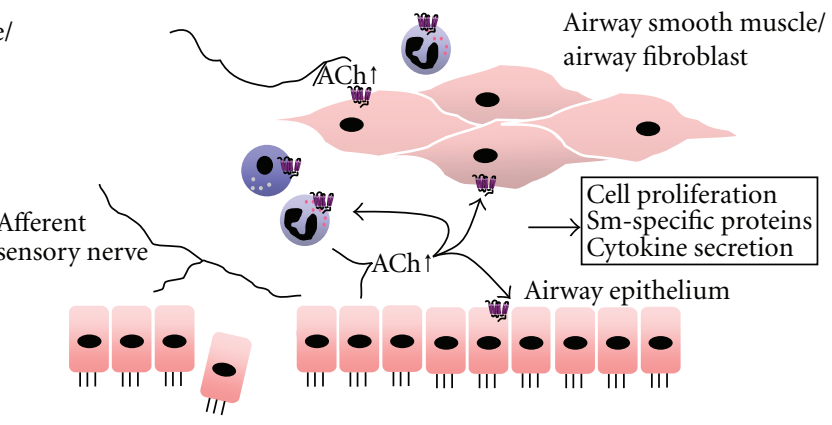

FIGURE 1: Muscarinic receptor regulation of airway inflammation and remodelling. In healthy airways, acetylcholine release from neuronal and nonneuronal origins release are limited. However, in response to environmental factors such as allergen or smoke, acetylcholine release is enhanced, which cooperates with proinflammatory cytokines and growth factors to induce airway smooth muscle and fibroblast cell responses including cell proliferation, smooth-muscle-specific protein expression, and the synthesis of chemokines and cytokines. As such, acetylcholine by acting on muscarinic receptors may contribute to both acute and chronic aspects of obstructive airways disease. Nicotinic receptors are expressed by airway structural cells and inhibit inflammatory cell activation; however, their role in regulating airway remodelling is largely unknown.

the airway smooth muscle itself. These mechanisms may promote airway inflammation and remodelling, including airway smooth muscle thickening.

\subsection{Indirect Effects: Acetylcholine as a Proinflammatory} Mediator. Airway inflammation in asthma and COPD likely plays an important role in the development of airway hyperresponsiveness and in the development of structural changes in the airway wall including increased airway smooth muscle mass. Inflammatory cells secrete cytokines and growth factors that induce a proliferative stimulus in airway smooth muscle cells (e.g., EGF, PDGF, and TGF- $\beta$ ) that may be amplified by the actions of acetylcholine as outlined above [56]. Moreover, acetylcholine, either from neuronal or nonneuronal origin, may regulate inflammatory cell responses in these diseases that explain the beneficial effects of anticholinergics on airway smooth muscle thickening [2] .

The anticholinergic agent tiotropium bromide prevented allergen-induced airway eosinophilia in guinea pigs, indicating that muscarinic receptor signalling supports airway eosinophilia [39]. It has been demonstrated that muscarinic $\mathrm{M}_{3}$ and $\mathrm{M}_{4}$ receptors are expressed in human and guinea pig eosinophils; human eosinophils also appear to express the muscarinic $M_{5}$ receptor subtype [57]. However, Verbout et al. found an inhibitory effect of these muscarinic receptors on eosinophil activation [58]. Atropine, a nonselective muscarinic receptor antagonist, significantly potentiated antigeninduced eosinophil activation and airway hyperreactivity by increasing major basic protein deposition in the airways [58]. The inhibitory effect of muscarinic recepors on eosinophil activation in antigen-challenged animals is mediated by their suppressive effect on excitatory nerve growth factor (NGF) pathway [59]. The effect of muscarinic receptors on airway structural cells (epithelial cells, fibroblasts, airway smooth muscle cells) as outlined above may account for this discrepancy as proinflammatory cytokine production by these cells, including the release of eosinophil chemotactic activity, is enhanced by muscarinic receptor stimulation.
Muscarinic receptors are also expressed by macrophages and neutrophils and appear to play an important proinflammatory role in these cells. Muscarinic $\mathrm{M}_{3}$ and $\mathrm{M}_{5}$ receptors are expressed by macrophages, and muscarinic receptor agonists, such as carbachol, induce an increase in intracellular calcium and promote chemotaxis of these cells [60]. Alveolar macrophages also appear to express muscarinic $\mathrm{M}_{1}, \mathrm{M}_{2}$, and $\mathrm{M}_{3}$ receptor subtypes [13]. Stimulation by acetylcholine of these cells induces the release of leukotriene $\mathrm{B}_{4}$, which promotes neutrophil chemotaxis. This contention is in agreement with a study showing that, in bovine alveolar macrophages, muscarinic $M_{3}$ receptors induce the release of leukotriene $B_{4}[61]$. Furthermore, it was recently shown that human alveolar macrophages respond to acetylcholine with the release of chemotactic activity for granulocytes, an effect likely involving leukotriene $B_{4}$ release [47]. The anticholinergic agent tiotropium suppressed the secretion of leukotriene $\mathrm{B}_{4}$ by more than $70 \%$ after acetylcholine stimulation [47].

Treatment with tiotropium bromide significantly reduced airway inflammation and the Th2 cytokine production in bronchoalveolar lavage fluid (BALF) in both acute and chronic models of asthma. The levels of TGFB1 in BALF, the goblet cell metaplasia, thickness of airway smooth muscle, and airway fibrosis were all significantly decreased in tiotropium bromide-treated mice as well [62]. Tiotropium also concentration-dependently inhibited neutrophilic inflammation in response to cigarette smoke. Furthermore, the cigarette-smoke-induced pulmonary release of leukotriene $\mathrm{B}(4)$, interleukin-6, keratinocyte-derived chemokine, monocyte chemotactic protein- 1 , macrophage inflammatory protein- 1 alpha and -2 , and tumour necrosis factor alpha was dose-dependently reduced in murine model of COPD [63]. Neutrophil-elastase-induced goblet cell hyperplasia and gastrointestinal reflux-induced pulmonary inflammation can also be prevented by tiotropium treatment $[64,65]$. These findings collectively indicate that acetylcholine, for example, nonneuronal acetylcholine derived 
from the inflammatory cells themselves, promotes inflammatory responses in the airways via muscarinic receptors.

\section{The Nicotinic Receptor: Acetylcholine as an Anti-Inflammatory Mediator}

The airway smooth muscle also expresses nicotinic receptors including the $\alpha 3$ and $\alpha 7$ nicotinic receptor subtypes [6]. The role of the nicotinic receptor in airway smooth muscle is currently largely unknown. However, in sharp contrast to the proinflammatory role of muscarinic receptor stimulation, nicotinic receptors appear to play an important antiinflammatory role in many cell types and organs. Nicotinic receptors are found in the airways on parasympathetic nerves, macrophages, eosinophils, neutrophils, mast cells [66-70], lymphocytes [71-73], airway smooth muscle cells [74], epithelial cells [75], and fibroblasts [76].

Acetylcholine from neuronal or nonneuronal origin can induce an anti-inflammatory effect via $\alpha 7$ nicotinic receptors in various models of acute inflammation [77, 78]. This is also established in models of pulmonary inflammation including a mouse model of hypersensitivity pneumonitis [79], asthma $[80,81]$, and inflammation following influenza infection $[82,83]$. These in vivo findings are supported by in vitro findings showing that stimulation of the $\alpha 7$ nicotinic receptor in murine macrophage cell lines results in inhibition of LPS-induced TNF and HMGB1 release [84-86]. Moreover, acetylcholine and nicotine receptor agonists exert a strong inhibitory effect on the release of TNF- $\alpha$ and other cytokines such as IL-6, IL- $1 \beta$, IL-12, IL-18, and IFN- $\gamma$ without affecting the production of anti-inflammatory cytokines although in some cases upregulation of IL-10 production is observed $[79,87-89]$. Acetylcholine anti-inflammatory properties are regulated by $\alpha 7$ nicotinic receptor on macrophages, because macrophages from $\alpha 7$-subunit-nicotinic-receptor-deficient mice failed to show inhibition of TNF- $\alpha$ release [66]. Local administration of GTS-21 (a selective $\alpha 7$ cholinergic receptor agonist) also inhibits TNF- $\alpha$ release in the mouse lung during LPS-induced inflammation [90].

In addition to exerting anti-inflammatory effects on macrophages, activation of $\alpha 7$ nicotinic receptors on endothelial cells inhibits TNF- $\alpha$-induced expression of intercellular adhesion molecule-1 and chemokines IL-8, RANTES (regulated on activation, normal $\mathrm{T}$ cell expressed and secreted), and monocyte chemoattractant protein-1 [91], thereby preventing migration of inflammatory cells from the blood to the tissues. Systemic administration of nicotine or a selective $\alpha 7$ agonist also attenuates acid-induced lung injury by reducing TNF- $\alpha$ and MIP-2 concentrations and by reducing neutrophil accumulation in the airspaces of the lung in rats, resulting in decreased pulmonary oedema and pulmonary inflammation [67]. Paradoxically, profibrotic, and proinflammatory effects of nicotine have also been reported, as nicotine appears to promote fibronectin deposition by fibroblasts [92]. Nonetheless, most reports point to an anti-inflammatory and antiremodelling role for the $\alpha 7$ nicotinic receptor. Thus, nicotinic agonists, including acetylcholine, can limit cytokine release and tissue inflammation. It was recently shown that $\alpha 7$ nicotinic receptors stimulation of alveolar macrophages and neutrophils also reduced chemokine production including MIP-2, transalveolar neutrophil migration, and LPS- and E. coli-induced acute lung injury in the airways of mice [67]. Collectively, these data indicate that acetylcholine may exert potent antiinflammatory effects in the lungs, primarily via $\alpha 7$ nicotinic receptors. Although expressed by airway smooth muscle, the role of the $\alpha 7$ nicotinic receptor is currently unknown. Clearly, experiments to identify the role of the $\alpha 7$ nicotinic receptor in airway smooth muscle, including its role in remodelling and in the immunomodulatory function of airway smooth muscle, are warranted.

\section{Conclusion}

Acetylcholine is the predominant parasympathetic neurotransmitter in the airways and an autocrine or paracrine hormone. Many structural and inflammatory cells, notably the airway epithelium, express and secrete acetylcholine and respond to acetylcholine (either neuronal or nonneuronal) via muscarinic and nicotinic receptors. The airway smooth muscle is of major importance to the physiological and pathophysiological actions of acetylcholine, which induces bronchoconstriction, airway smooth muscle thickening, and the modulation of cytokine and chemokine production by these cells (Figure 1). Additionally, muscarinic receptors regulate proinflammatory and remodelling responses of fibroblasts and airway epithelial cells and promote the release of leukotriene $\mathrm{B}_{4}$ and other chemotactic mediators from macrophages and epithelial cells, resulting in eosinophil and neutrophil chemotactic activity.

In contrast to this proinflammatory role, nicotinic receptors expressed by inflammatory cells and structural cells exert potent anti-inflammatory effects, in which the $\alpha 7$ nicotinic receptor appears to play a central role. This receptor subtype can be targeted both by neuronal and nonneuronal acetylcholine and may present a useful therapeutic target for treatment. The role of the $\alpha 7$ nicotinic receptor in airway smooth muscle, and in airway remodelling in asthma and COPD, is currently largely unknown but clearly warrants future investigation. In addition, it is essential to design future studies to identify the (patho)physiological basis for the clear discrepancy between nicotinic and muscarinic receptor subtypes in the regulation of inflammation and remodelling.

Clearly, the airway cholinergic system holds excellent therapeutic potential. Muscarinic receptor antagonists, currently widely used as bronchodilators for the treatment of COPD, may have beneficial anti-inflammatory and antiremodelling effects. Although direct evidence for this assumption is lacking in asthma and COPD patients, treatment with the anticholinergic agent tiotropium reduces exacerbation frequency in COPD patients and reduces lung function decline in GOLD stage II COPD patients [93, 94]. These clinical findings are consistent with anti-inflammatory and remodelling effects of tiotropium, but proof for this hypothesis still needs to be obtained. In addition, the antiinflammatory effects of the $\alpha 7$ nicotinic receptor suggest 
that agonists for this receptor subtype are a strategy worth pursuing for the treatment of asthma and COPD.

\section{Acknowledgment}

R. Gosens is supported by a ZonMW Veni Grant from the Netherlands Organization of Scientific Research (916.86.036).

\section{References}

[1] K. E. Belmonte, "Cholinergic pathways in the lungs and anticholinergic therapy for chronic obstructive pulmonary disease," Proceedings of the American Thoracic Society, vol. 2, no. 4, pp. 297-304, 2005.

[2] R. Gosens, J. Zaagsma, H. Meurs, and A. J. Halayko, "Muscarinic receptor signaling in the pathophysiology of asthma and COPD," Respiratory Research, vol. 7, article 73, 2006.

[3] K. Racké and S. Matthiesen, "The airway cholinergic system: physiology and pharmacology," Pulmonary Pharmacology and Therapeutics, vol. 17, no. 4, pp. 181-198, 2004.

[4] K. Racké, U. R. Juergens, and S. Matthiesen, "Control by cholinergic mechanisms," European Journal of Pharmacology, vol. 533, no. 1-3, pp. 57-68, 2006.

[5] B. J. Proskocil, H. S. Sekhon, Y. Jia et al., "Acetylcholine is an autocrine or paracrine hormone synthesized and secreted by airway bronchial epithelial cells," Endocrinology, vol. 145, no. 5, pp. 2498-2506, 2004.

[6] I. Wessler and C. J. Kirkpatrick, "Acetylcholine beyond neurons: the non-neuronal cholinergic system in humans," British Journal of Pharmacology, vol. 154, no. 8, pp. 1558-1571, 2008.

[7] I. K. Wessler and C. J. Kirkpatrick, "The non-neuronal cholinergic system: an emerging drug target in the airways," Pulmonary Pharmacology and Therapeutics, vol. 14, no. 6, pp. 423-434, 2001.

[8] J. Wess, R. M. Eglen, and D. Gautam, "Muscarinic acetylcholine receptors: mutant mice provide new insights for drug development," Nature Reviews Drug Discovery, vol. 6, no. 9, pp. 721-733, 2007.

[9] W. Kummer, K. S. Lips, and U. Pfeil, "The epithelial cholinergic system of the airways," Histochemistry and Cell Biology, vol. 130, no. 2, pp. 219-234, 2008.

[10] K. S. Lips, C. Volk, B. M. Schmitt et al., "Polyspecific cation transporters mediate luminal release of acetylcholine from bronchial epithelium," American Journal of Respiratory Cell and Molecular Biology, vol. 33, no. 1, pp. 79-88, 2005.

[11] W. Kummer, S. Wiegand, S. Akinci et al., "Role of acetylcholine and polyspecific cation transporters in serotonin-induced bronchoconstriction in the mouse," Respiratory Research, vol. 7, article 65, 2006.

[12] W. Kummer, S. Wiegand, S. Akinci et al., "Role of acetylcholine and muscarinic receptors in serotonin-induced bronchoconstriction in the mouse," Journal of Molecular Neuroscience, vol. 30, no. 1-2, pp. 67-68, 2006.

[13] M. Profita, R. Di Giorgi, A. Sala et al., "Muscarinic receptors, leukotriene B4 production and neutrophilic inflammation in COPD patients," Allergy, vol. 60, no. 11, pp. 1361-1369, 2005.

[14] M. Profita, A. Bonanno, L. Siena et al., "Smoke, choline acetyltransferase, muscarinic receptors, and fibroblast proliferation in chronic obstructive pulmonary disease," Journal of
Pharmacology and Experimental Therapeutics, vol. 329, no. 2, pp. 753-763, 2009.

[15] A. F. Roffel, C. R. S. Elzinga, R. G. M. van Amsterdam, R. A. de Zeeuw, and J. Zaagsma, "Muscarinic M2 receptor in bovine tracheal smooth muscle: discrepancies between binding and function," European Journal of Pharmacology, vol. 153, no. 1, pp. 73-82, 1988.

[16] H. Meurs, A. F. Roffel, J. B. Postema et al., "Evidence for a direct relationship between phosphoinositide metabolism and airway smooth muscle contraction induced by muscarinic agonists," European Journal of Pharmacology, vol. 156, no. 2, pp. 271-274, 1988.

[17] A. F. Roffel, H. Meurs, C. R. S. Elzinga, and J. Zaagsma, "Characterization of the muscarinic receptor subtype involved in phosphinositide metabolism in bovine tracheal smooth muscle," British Journal of Pharmacology, vol. 99, no. 2, pp. 293-296, 1990.

[18] J. T. Fisher, S. G. Vincent, J. Gomeza, M. Yamada, and J. Wess, "Loss of vagally mediated bradycardia and bronchoconstriction in mice lacking M2 or M3 muscarinic acetylcholine receptors," The FASEB Journal, vol. 18, no. 6, pp. 711-713, 2004.

[19] C. K. Billington, K. C. Kong, R. Bhattacharyya et al., "Cooperative regulation of p70S6 kinase by receptor tyrosine kinases and $\mathrm{G}$ protein-coupled receptors augments airway smooth muscle growth," Biochemistry, vol. 44, no. 44, pp. 1459514605, 2005.

[20] V. P. Krymskaya, M. J. Orsini, A. J. Eszterhas et al., "Mechanisms of proliferation synergy by receptor tyrosine kinase and $\mathrm{G}$ protein-coupled receptor activation in human airway smooth muscle," American Journal of Respiratory Cell and Molecular Biology, vol. 23, no. 4, pp. 546-554, 2000.

[21] K. C. Kong, C. K. Billington, U. Gandhi, R. A. Panettieri, and R. B. Penn, "Cooperative mitogenic signaling by G proteincoupled receptors and growth factors is dependent on $\mathrm{G}_{q / 11}$," The FASEB Journal, vol. 20, no. 9, pp. 1558-1560, 2006.

[22] R. Gosens, S. A. Nelemans, M. M. Grootte Bromhaar, S. McKay, J. Zaagsma, and H. Meurs, "Muscarinic M3-receptors mediate cholinergic synergism of mitogenesis in airway smooth muscle," American Journal of Respiratory Cell and Molecular Biology, vol. 28, no. 2, pp. 257-262, 2003.

[23] R. Gosens, G. Dueck, E. Rector et al., "Cooperative regulation of GSK-3 by muscarinic and PDGF receptors is associated with airway myocyte proliferation," American Journal of Physiology, vol. 293, no. 5, pp. L1348-L1358, 2007.

[24] J. K. Bentley, H. Deng, M. J. Linn et al., "Airway smooth muscle hyperplasia and hypertrophy correlate with glycogen synthase kinase-3(beta) phosphorylation in a mouse model of asthma," American Journal of Physiology, vol. 296, no. 2, pp. L176-L184, 2009.

[25] H. Deng, G. A. Dokshin, J. Lei et al., "Inhibition of glycogen synthase kinase-3 $\beta$ is sufficient for airway smooth muscle hypertrophy," Journal of Biological Chemistry, vol. 283, no. 15, pp. 10198-10207, 2008.

[26] R. O. Nunes, M. Schmidt, G. Dueck et al., "GSK-3/ $\beta$-catenin signaling axis in airway smooth muscle: role in mitogenic signaling," American Journal of Physiology, vol. 294, no. 6, pp. L1110-L1118, 2008.

[27] D. J. Tschumperlin, G. Dal, I. V. Maly et al., "Mechanotransduction through growth-factor shedding into the extracellular space," Nature, vol. 429, no. 6987, pp. 83-86, 2004.

[28] C. L. Grainge, L. C.K. Lau, J. A. Ward et al., "Effect of bronchoconstriction on airway remodeling in asthma," The 
New England Journal of Medicine, vol. 364, no. 21, pp. 2006 2015, 2011.

[29] N. A. Hasaneen, S. Zucker, J. Cao, C. Chiarelli, R. A. Panettieri, and H. D. Foda, "Cyclic mechanical strain-induced proliferation and migration of human airway smooth muscle cells: role of EMMPRIN and MMPs," The FASEB Journal, vol. 19, no. 11, pp. 1507-1509, 2005.

[30] P. G. Smith, K. E. Janiga, and M. C. Bruce, "Strain increases airway smooth muscle cell proliferation," American Journal of Respiratory Cell and Molecular Biology, vol. 10, no. 1, pp. 8590, 1994.

[31] P. G. Smith, T. Tokui, and M. Ikebe, "Mechanical strain increases contractile enzyme activity in cultured airway smooth muscle cells," American Journal of Physiology, vol. 268, no. 6, pp. L999-L1005, 1995.

[32] N. J. Fairbank, S. C. Connolly, J. D. MacKinnon, K. Wehry, L. Deng, and G. N. Maksym, "Airway smooth muscle cell tone amplifies contractile function in the presence of chronic cyclic strain," American Journal of Physiology, vol. 295, no. 3, pp. L479-L488, 2008.

[33] O. Tliba and R. A. Panettieri, "Noncontractile functions of airway smooth muscle cells in asthma," Annual Review of Physiology, vol. 71, pp. 509-535, 2009.

[34] S. Zuyderduyn, M. B. Sukkar, A. Fust, S. Dhaliwal, and J. K. Burgess, "Treating asthma means treating airway smooth muscle cells," European Respiratory Journal, vol. 32, no. 2, pp. 265-274, 2008.

[35] J. Kanefsky, M. Lenburg, and C. M. Hai, “Cholinergic receptor and cyclic stretch-mediated inflammatory gene expression in intact ASM," American Journal of Respiratory Cell and Molecular Biology, vol. 34, no. 4, pp. 417-425, 2006.

[36] R. Gosens, D. Rieks, H. Meurs et al., "Muscarinic M3 receptor stimulation increases cigarette smoke-induced IL-8 secretion by human airway smooth muscle cells," European Respiratory Journal, vol. 34, no. 6, pp. 1436-1443, 2009.

[37] T. A. Oenema, S. Kolahian, J. E. Nanninga et al., "Proinflammatory mechanisms of muscarinic receptor stimulation in airway smooth muscle," Respiratory Research, vol. 11, article 130, 2010.

[38] R. Gosens, I. S. T. Bos, J. Zaagsma, and H. Meurs, "Protective effects of tiotropium bromide in the progression of airway smooth muscle remodeling," American Journal of Respiratory and Critical Care Medicine, vol. 171, no. 10, pp. 1096-1102, 2005.

[39] I. S. T. Bos, R. Gosens, A. B. Zuidhof et al., "Inhibition of allergen-induced airway remodelling by tiotropium and budesonide: a comparison," European Respiratory Journal, vol. 30, no. 4, pp. 653-661, 2007.

[40] B. G. J. Dekkers, H. Maarsingh, H. Meurs, and R. Gosens, "Airway structural components drive airway smooth muscle remodeling in asthma," Proceedings of the American Thoracic Society, vol. 6, no. 8, pp. 683-692, 2009.

[41] D. S. Postma and W. Timens, "Remodeling in asthma and chronic obstructive pulmonary disease," Proceedings of the American Thoracic Society, vol. 3, no. 5, pp. 434-439, 2006.

[42] S. Matthiesen, A. Bahulayan, S. Kempkens et al., "Muscarinic receptors mediate stimulation of human lung fibroblast proliferation," American Journal of Respiratory Cell and Molecular Biology, vol. 35, no. 6, pp. 621-627, 2006.

[43] S. Matthiesen, A. Bahulayan, O. Holz, and K. Racké, "MAPK pathway mediates muscarinic receptor-induced human lung fibroblast proliferation," Life Sciences, vol. 80, no. 24-25, pp. 2259-2262, 2007.
[44] M. P. Pieper, N. I. Chaudhary, and J. E. Park, "Acetylcholineinduced proliferation of fibroblasts and myofibroblasts in vitro is inhibited by tiotropium bromide," Life Sciences, vol. 80, no. 24-25, pp. 2270-2273, 2007.

[45] S. Haag, S. Matthiesen, U. R. Juergens, and K. Racké, "Muscarinic receptors mediate stimulation of collagen synthesis in human lung fibroblasts," European Respiratory Journal, vol. 32, no. 3, pp. 555-562, 2008.

[46] T. Pera, A. Zuidhof, J. Valadas et al., "Tiotropium inhibits pulmonary inflammation and remodelling in a guinea pig model of COPD," European Respiratory Journal, vol. 38, no. 4, pp. 789-796, 2011.

[47] F. Bühling, N. Lieder, U. C. Kühlmann, N. Waldburg, and T. Welte, "Tiotropium suppresses acetylcholine-induced release of chemotactic mediators in vitro," Respiratory Medicine, vol. 101, no. 11, pp. 2386-2394, 2007.

[48] K. Asano, Y. Shikama, Y. Shibuya et al., "Suppressive activity of tiotropium bromide on matrix metalloproteinase production from lung fibroblasts in vitro," International Journal of Chronic Obstructive Pulmonary Disease, vol. 3, no. 4, pp. 781-790, 2008.

[49] G. Jositsch, T. Papadakis, R. V. Haberberger, M. Wolff, J. Wess, and W. Kummer, "Suitability of muscarinic acetylcholine receptor antibodies for immunohistochemistry evaluated on tissue sections of receptor gene-deficient mice," NaunynSchmiedeberg's Archives of Pharmacology, vol. 379, no. 4, pp. 389-395, 2009.

[50] H. Klapproth, T. Reinheimer, J. Metzen et al., "Non-neuronal acetylcholine, a signalling molecule synthezised by surface cells of rat and man," Naunyn-Schmiedeberg's Archives of Pharmacology, vol. 355, no. 4, pp. 515-523, 1997.

[51] J. Metzen, F. Bittinger, C. J. Kirkpatrick, H. Kilbinger, and I. Wessler, "Proliferative effect of acetylcholine on rat trachea epithelial cells is mediated by nicotinic receptors and muscarinic receptors of the M1-subtype," Life Sciences, vol. 72, no. 18-19, pp. 2075-2080, 2003.

[52] S. Koyama, S. I. Rennard, and R. A. Robbins, "Acetylcholine stimulates bronchial epithelial cells to release neutrophil and monocyte chemotactic activity," American Journal of Physiology, vol. 262, no. 4, pp. L466-L471, 1992.

[53] S. Koyama, E. Sato, H. Nomura, K. Kubo, S. Nagai, and T. Izumi, "Acetylcholine and substance P stimulate bronchial epithelial cells to release eosinophil chemotactic activity," Journal of Applied Physiology, vol. 84, no. 5, pp. 1528-1534, 1998.

[54] G. Brunn, I. Wessler, and K. Racke, "Mucosa-dependent muscarinic liberation of prostaglandins from rat isolated trachea," British Journal of Pharmacology, vol. 116, no. 3, pp. 1991-1998, 1995.

[55] M. Profita, A. Bonanno, L. Siena et al., "Acetylcholine mediates the release of IL-8 in human bronchial epithelial cells by a NFkB/ERK-dependent mechanism," European Journal of Pharmacology, vol. 582, no. 1-3, pp. 145-153, 2008.

[56] R. Gosens, S. S. Roscioni, B. G. J. Dekkers et al., "Pharmacology of airway smooth muscle proliferation," European Journal of Pharmacology, vol. 585, no. 2-3, pp. 385-397, 2008.

[57] N. G. Verbout, J. K. Lorton, D. B. Jacoby, and A. D. Fryer, "A functional role for muscarinic receptors on eosinophils in the airways," Proceedings of the American Thoracic Society, vol. 3, p. A587, 2006.

[58] N. G. Verbout, J. K. Lorton, D. B. Jacoby, and A. D. Fryer, "Atropine pretreatment enhances airway hyperreactivity 
in antigen-challenged guinea pigs through an eosinophildependent mechanism," American Journal of Physiology, vol. 292, no. 5, pp. L1126-L1135, 2007.

[59] N. G. Verbout, D. B. Jacoby, G. J. Gleich, and A. D. Fryer, "Atropine-enhanced, antigen challenge-induced airway hyperreactivity in guinea pigs is mediated by eosinophils and nerve growth factor," American Journal of Physiology, vol. 297, no. 2, pp. L228-L237, 2009.

[60] Y. Mita, K. Dobashi, K. Suzuki, M. Mori, and T. Nakazawa, "Induction of muscarinic receptor subtypes in monocytic/macrophagic cells differentiated from EoL-1 cells," European Journal of Pharmacology, vol. 297, no. 1-2, pp. 121-127, 1996.

[61] E. Sato, S. Koyama, Y. Okubo, K. Kubo, and M. Sekiguchi, "Acetylcholine stimulates alveolar macrophages to release inflammatory cell chemotactic activity," American Journal of Physiology, vol. 274, no. 6, pp. L970-L979, 1998.

[62] S. Ohta, N. Oda, T. Yokoe et al., "Effect of tiotropium bromide on airway inflammation and remodelling in a mouse model of asthma," Clinical and Experimental Allergy, vol. 40, no. 8, pp. 1266-1275, 2010.

[63] L. Wollin and M. P. Pieper, "Tiotropium bromide exerts antiinflammatory activity in a cigarette smoke mouse model of COPD," Pulmonary Pharmacology and Therapeutics, vol. 23, no. 4, pp. 345-354, 2010.

[64] N. Arai, M. Kondo, T. Izumo, J. Tamaoki, and A. Nagai, "Inhibition of neutrophil elastase-induced goblet cell metaplasia by tiotropium in mice," European Respiratory Journal, vol. 35, no. 5, pp. 1164-1171, 2010.

[65] Y. Cui, P. Devillier, X. Kuang et al., "Tiotropium reduction of lung inflammation in a model of chronic gastro-oesophageal reflux," European Respiratory Journal, vol. 35, no. 6, pp. 13701376, 2010.

[66] H. Wang, M. Yu, M. Ochani et al., "Nicotinic acetylcholine receptor $\alpha 7$ subunit is an essential regulator of inflammation," Nature, vol. 421, no. 6921, pp. 384-388, 2003.

[67] X. Su, W. L. Jae, Z. A. Matthay et al., "Activation of the $\alpha 7 \mathrm{nAChR}$ reduces acid-induced acute lung injury in mice and rats," American Journal of Respiratory Cell and Molecular Biology, vol. 37, no. 2, pp. 186-192, 2007.

[68] M. R. Blanchet, A. Langlois, E. Israël-Assayag et al., "Modulation of eosinophil activation in vitro by a nicotinic receptor agonist," Journal of Leukocyte Biology, vol. 81, no. 5, pp. 12451251, 2007.

[69] S. Iho, Y. Tanaka, R. Takauji et al., "Nicotine induces human neutrophils to produce IL-8 through the generation of peroxynitrite and subsequent activation of NF- $\kappa \mathrm{B}$," Journal of Leukocyte Biology, vol. 74, no. 5, pp. 942-951, 2003.

[70] P. S. Sudheer, J. E. Hall, R. Donev, G. Read, A. Rowbottom, and P. E. Williams, "Nicotinic acetylcholine receptors on basophils and mast cells," Anaesthesia, vol. 61, no. 12, pp. 1170-1174, 2006.

[71] H. S. Sekhon, J. Yibing, R. Raab et al., "Prenatal nicotine increases pulmonary $\alpha 7$ nicotinic receptor expression and alters fetal lung development in monkeys," Journal of Clinical Investigation, vol. 103, no. 5, pp. 637-647, 1999.

[72] E. Battaglioli, C. Gotti, S. Terzano, A. Flora, F. Clementi, and D. Fornasari, "Expression and transcriptional regulation of the human $\alpha 3$ neuronal nicotinic receptor subunit in $\mathrm{T}$ lymphocyte cell lines," Journal of Neurochemistry, vol. 71, no. 3, pp. 1261-1270, 1998.

[73] K. Kawashima and T. Fujii, "Extraneuronal cholinergic system in lymphocytes," Pharmacology and Therapeutics, vol. 86, no. 1, pp. 29-48, 2000.
[74] G. Dorion, E. Israël-Assayag, M. J. Beaulieu, and Y. Cormier, "Effect of 1,1-dimethylphenyl 1,4-piperazinium on mouse tracheal smooth muscle responsiveness," American Journal of Physiology, vol. 288, no. 6, pp. L1139-L1145, 2005.

[75] A. D. J. Maus, E. F. R. Pereira, P. I. Karachunski et al., "Human and rodent bronchial epithelial cells express functional nicotinic acetylcholine receptors," Molecular Pharmacology, vol. 54, no. 5, pp. 779-788, 1998.

[76] J. Lahmouzi, F. Simain-Sato, M. P. Defresne et al., "Effect of nicotine on rat gingival fibroblasts in vitro," Connective Tissue Research, vol. 41, no. 1, pp. 69-80, 2000.

[77] V. A. Pavlov and K. J. Tracey, "The cholinergic antiinflammatory pathway," Brain, Behavior, and Immunity, vol. 19, no. 6, pp. 493-499, 2005.

[78] W. J. de Jonge and L. Ulloa, "The alpha7 nicotinic acetylcholine receptor as a pharmacological target for inflammation," British Journal of Pharmacology, vol. 151, no. 7, pp. 915929, 2007.

[79] M. R. Blanchet, E. Israël-Assayag, and Y. Cormier, "Inhibitory effect of nicotine on experimental hypersensitivity pneumonitis in vivo and in vitro," American Journal of Respiratory and Critical Care Medicine, vol. 169, no. 8, pp. 903-909, 2004.

[80] M. R. Blanchet, E. Israël-Assayag, and Y. Cormier, "Modulation of airway inflammation and resistance in mice by a nicotinic receptor agonist," European Respiratory Journal, vol. 26, no. 1, pp. 21-27, 2005.

[81] N. C. Mishra, J. Rir-sima-ah, R. J. Langley et al., "Nicotine primarily suppresses lung Th2 but not goblet cell and muscle cell responses to allergens," Journal of Immunology, vol. 180, no. 11, pp. 7655-7663, 2008.

[82] R. Kalra, S. P. Singh, J. C. Pena-Philippides, R. J. Langley, S. Razani-Boroujerdi, and M. L. Sopori, "Immunosuppressive and anti-inflammatory effects of nicotine administered by patch in an animal model," Clinical and Diagnostic Laboratory Immunology, vol. 11, no. 3, pp. 563-568, 2004.

[83] S. Razani-Boroujerdi, S. P. Singh, C. Knall et al., "Chronic nicotine inhibits inflammation and promotes influenza infection," Cellular Immunology, vol. 230, no. 1, pp. 1-9, 2004.

[84] H. Wang, H. Liao, M. Ochani et al., "Cholinergic agonists inhibit HMGB1 release and improve survival in experimental sepsis," Nature Medicine, vol. 10, no. 11, pp. 1216-1221, 2004.

[85] V. A. Pavlov, M. Ochani, L. H. Yang et al., "Selective $\alpha 7$ nicotinic acetylcholine receptor agonist GTS-21 improves survival in murine endotoxemia and severe sepsis," Critical Care Medicine, vol. 35, no. 4, pp. 1139-1144, 2007.

[86] W. R. Parrish, M. Rosas-Ballina, M. Gallowitsch-Puerta et al., "Modulation of TNF release by choline requires $\alpha 7$ subunit nicotinic acetylcholine receptor-mediated signaling," Molecular Medicine, vol. 14, no. 9-10, pp. 567-574, 2008.

[87] L. V. Borovikova, S. Ivanova, M. Zhang et al., "Vagus nerve stimulation attenuates the systemic inflammatory response to endotoxin," Nature, vol. 405, no. 6785, pp. 458-462, 2000.

[88] K. Matsunaga, T. W. Klein, H. Friedman, and Y. Yamamoto, "Involvement of nicotinic acetylcholine receptors in suppression of antimicrobial activity and cytokine responses of alveolar macrophages to Legionella pneumophila infection by nicotine," Journal of Immunology, vol. 167, no. 11, pp. 65186524, 2001.

[89] A. I. Chernyavsky, J. Arredondo, M. Skok, and S. A. Grando, "Auto/paracrine control of inflammatory cytokines by acetylcholine in macrophage-like U937 cells through nicotinic receptors," International Immunopharmacology, vol. 10, no. 3, pp. 308-315, 2010. 
[90] I. A. J. Giebelen, D. J. van Westerloo, G. J. LaRosa, A. F. de Vos, and T. van der Poll, "Local stimulation of $\alpha 7$ cholinergic receptors inhibits LPS-induced TNF- $\alpha$ release in the mouse lung," Shock, vol. 28, no. 6, pp. 700-703, 2007.

[91] R. W. Saeed, S. Varma, T. Peng-Nemeroff et al., "Cholinergic stimulation blocks endothelial cell activation and leukocyte recruitment during inflammation," Journal of Experimental Medicine, vol. 201, no. 7, pp. 1113-1123, 2005.

[92] J. Roman, J. D. Ritzenthaler, A. Gil-Acosta, H. N. Rivera, and S. Roser-Page, "Nicotine and fibronectin, expression in lung fibroblasts: implications for tobacco-related lung tissue remodeling," The FASEB Journal, vol. 18, no. 12, pp. 14361438, 2004.

[93] M. Decramer, B. Celli, S. Kesten, T. Lystig, S. Mehra, and D. P. Tashkin, "Effect of tiotropium on outcomes in patients with moderate chronic obstructive pulmonary disease (UPLIFT): a prespecified subgroup analysis of a randomised controlled trial," The Lancet, vol. 374, no. 9696, pp. 1171-1178, 2009.

[94] D. P. Tashkin, B. Celli, S. Senn et al., "A 4-year trial of tiotropium in chronic obstructive pulmonary disease," The New England Journal of Medicine, vol. 359, no. 15, pp. 15431554, 2008. 


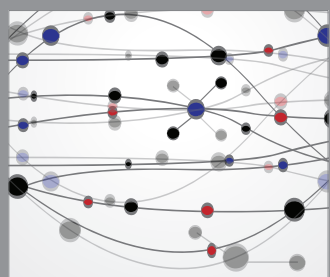

The Scientific World Journal
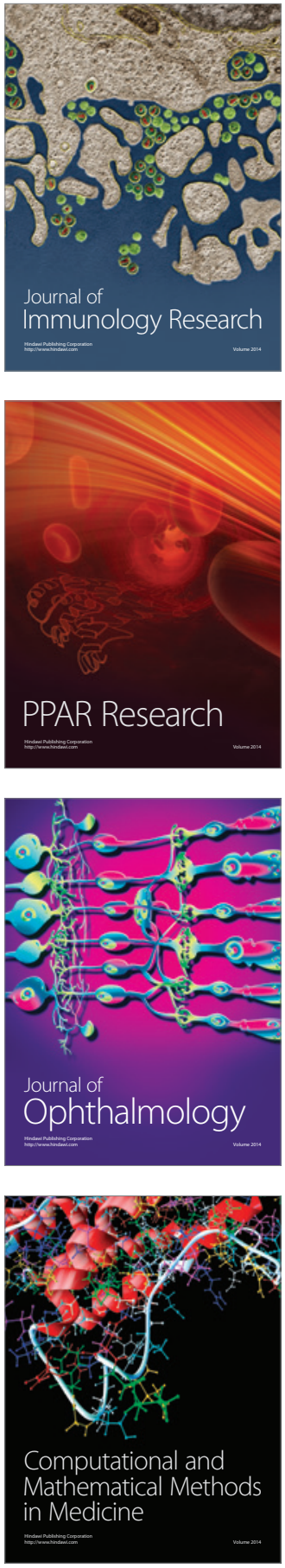

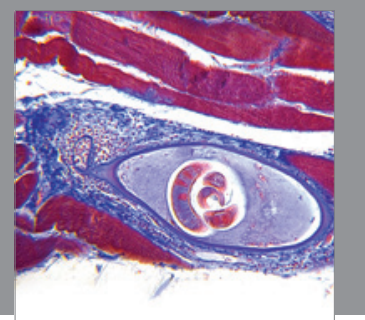

Gastroenterology

Research and Practice
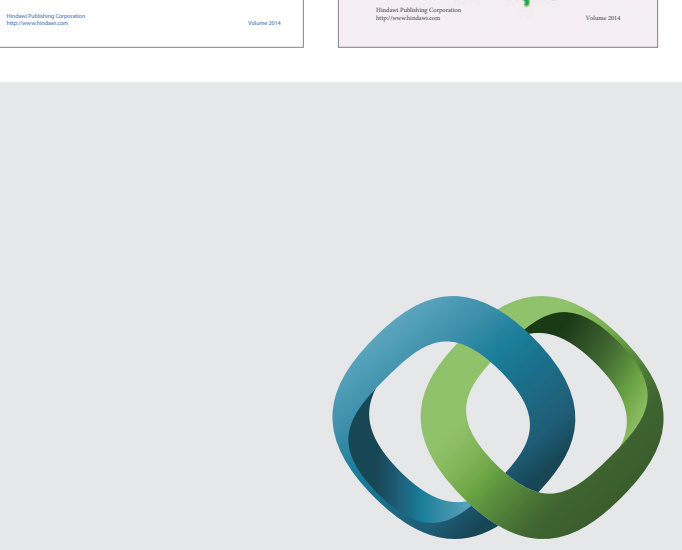

\section{Hindawi}

Submit your manuscripts at

http://www.hindawi.com
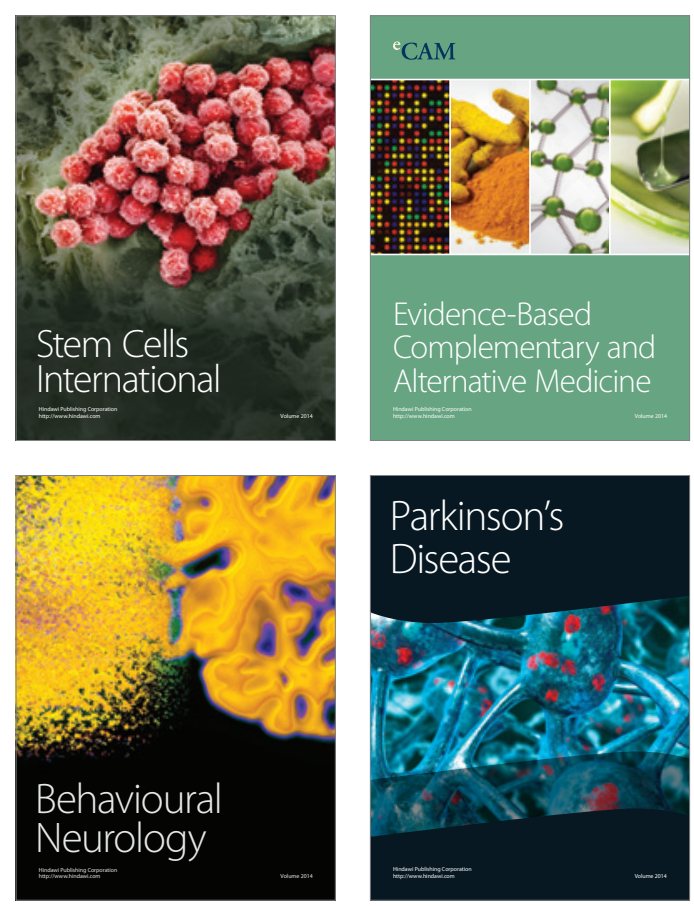

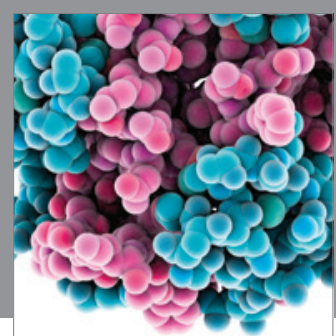

Journal of
Diabetes Research

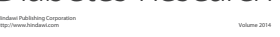

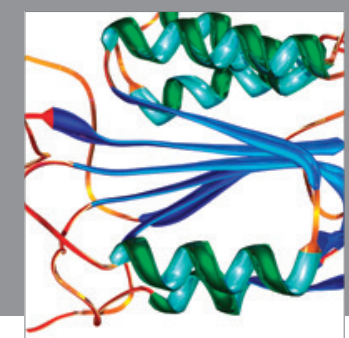

Disease Markers
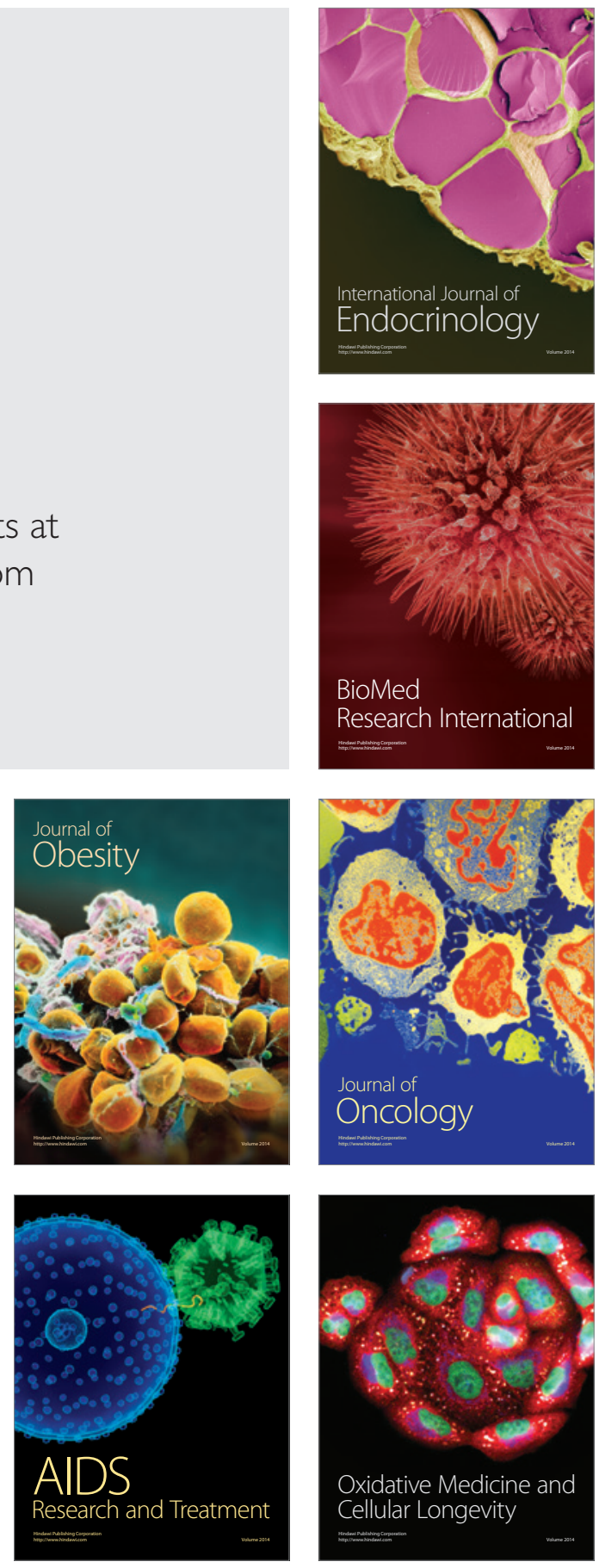